\title{
Ethnic disparities in influenza vaccination in Canada
}

\author{
Bradford D. Gessner MD MPH
}

See related research article by Quach and colleagues on page 1673 and at www.cmaj.ca/lookup/doi/10.1503/cmaj.111628

I $\mathrm{n}$ this issue of CMAJ, Quach and colleagues present the results of a large cross-sectional Canadian study that identified significant ethnic disparities in influenza vaccination. ${ }^{1}$ Most importantly, after adjusting for sociodemographic variables (e.g., education, use of health services, household income, length of time in Canada and access to universal influenza vaccination programs), the authors found that people who self-identified as white or black were less likely to get the vaccination than people of Asian or Aboriginal descent. However, are these data supported by other studies? If they are, what are the implications for public health?

At least three studies from the United States have found that non-Hispanic white people were more likely to receive the vaccination than black or Hispanic people. Two of these studies used national survey data but did not control for socioeconomic variables; ${ }^{2,3}$ the third study was more limited, but it included results adjusted for income, employment status, insurance status, age and sex. ${ }^{4}$ In agreement with Quach and colleagues, other studies have found that people of certain ethnicities were more likely to receive the vaccination for pandemic 2009 A (H1N1) influenza than white people. ${ }^{5}$ Although studies have consistently shown ethnic and racial disparities, the ethnic or racial groups at risk of lower vaccine coverage will vary depending on the social and epidemiologic contexts of a particular time and place.

What explanation might there be for these differences? The answer is likely primarily local context. In addition to the factors reported by Quach and colleagues, this may include access to vaccination programs, attitudes of local clinicians, access to the Internet, the importance of the antivaccine movement, media reporting and bias, actual or perceived prevalence of disease in a population, and assessment of individual risk. Local societal attributes may also play a key role: Bardenheier and coauthors recently documented that among residents of long-term care facilities in Michigan in the United States, coverage for influenza vaccination was lowest among residents - whether they were black or white - from facilities with the highest percentage of black residents. ${ }^{6}$
A number of studies have identified potential public health interventions that might improve uptake in vaccination programs - specifically for influenza. One Cochrane review evaluated the evidence base for patient reminder and recall systems to improve vaccination rates. ${ }^{7}$ The reminders or methods of recall used varied by study and included postcards, letters, and personal and autodial telephone calls; the target of the intervention also varied (patients or providers). Regardless of these nuances, the authors concluded that patient reminders and recalls were effective in all settings. In addition, specifically for influenza, this review showed the effectiveness of reminder/recall strategies across age groups. Among children, all 5 of the included studies reported a positive effect, with an improvement in coverage of up to 26 percentage points. Among adults, more than 20 studies were included; the impact on coverage varied more widely (from $-8 \%$ to $47 \%$ ), but mainly showed improvement.

A subsequent Cochrane review in 2010 focused on influenza vaccination rates among older adults and all published interventions. ${ }^{8}$ However, the authors identified only 13 relevant high-quality, randomized clinical trials, most of which involved patient or physician reminders. In general, patient reminders were effective, whereas physician reminders were not.

Risk factors for poor coverage suggest other potential interventions. These might include placing influenza vaccination centres more widely throughout the community, more concerted advocacy to counteract antivaccine messages and vaccine hesitancy, education targeting

\section{KEY POINTS}

- Although studies have consistently shown ethnic and racial disparities in vaccination coverage, the ethnic or racial groups at risk will vary depending on the social and epidemiologic contexts at a particular time and place.

- Ethnic and racial disparities in vaccination coverage likely reflect unmeasured confounders that are more appropriate targets for public health interventions.

- Public health interventions to improve the coverage of vaccination programs should define appropriate risk groups, identify evidencebased interventions, recognize the importance of local context and measure effectiveness over time. 
clinicians, developing a more effective vaccine with broader coverage and a longer duration of protection, and incentive systems. However, data are lacking to support such interventions.

In addition, before defining and evaluating interventions, it would be useful to more accurately identify the most appropriate target groups. For example, the World Health Organization (WHO) in 2005 identified 5 high-priority groups, 3 of which included elderly people: those in longterm care facilities, those with chronic conditions and those above a nationally defined age limit.' However, recent reviews have highlighted the weakness of data on vaccine effectiveness among people younger than 6 and older than 64 years of age (i.e., most target groups). ${ }^{10}$ Moreover, target groups may change: using data from the 2009 influenza A (H1N1) pandemic, the WHO may soon emphasize women who are pregnant as the group with the highest priority for vaccination.

As target groups change, the relevance of ethnic and racial disparities will likely also change. Dlugacz and coauthors documented that $34 \%$ of women in a 4-hospital study received vaccination against 2009 pandemic influenza A (H1N1). ${ }^{11}$ Coverage was low in all groups and varied almost 2 -fold by race, with $37 \%-40 \%$ coverage among Asian and white people, 30\% among Hispanic people and 23\% among all other ethnicities.

Finally, it is worth considering why we should care about racial or ethnic differences at all. Two situations come to mind. First, some jurisdictions may divide health care services by race; for example, in Alaska, Aboriginal people have access to the Alaska Native Health Care System, whereas non-Aboriginal Alaskans receive care through private or military providers. Second, some outcomes may cluster by race (a classic example being sickle cell disease among people of African descent). In both cases, public health providers and clinicians can tailor interventions and resources by race.

Other than in these 2 situations, the main usefulness of identifying racial and ethnic disparities is to show a need to search for the underlying causes that can then be the focus of public health interventions. The results of the study by Quach and colleagues, and their interpretation, changed substantially depending on whether one looked at the bivariate or multivariate analysis. This is an example of the importance of confounding by social and environmental variables in almost all associations between race and health outcomes. Similarly, the association reported by Quach and colleagues almost certainly represents residual confounding by unmeasured variables, such as cultural beliefs that may be shared across ethnicities.

Even if one could identify social or cultural factors that both influence vaccination coverage and highly segregate by race, it is not clear how this knowledge could be used. Historically, racebased health interventions have failed. One such example is the attempt to limit newborn hemoglobinopathy screening to black infants based on the high prevalence of sickle cell disease; screening was first expanded to Asian infants to cover those at risk for thalassemia and now includes all newborns. Furthermore, although race and ethnicity have never been particularly robust constructs for designing health interventions, they will become increasingly irrelevant with global population movements, such as travel and migration, and increasing numbers of multiracial people.

Rather than focus on race and ethnicity, I suggest a different approach. First, we should acknowledge that vaccination coverage for influenza is low in almost all groups, the optimal target groups are not yet well-defined, and target groups and risk factors for low coverage will vary with place and time. For these reasons, national and local public health agencies should develop interventions that address risk factors at the appropriate level and for all people. An integral component of interventions should be to measure their effectiveness over time and to accept that new approaches may be needed as contexts change.

\section{References}

1. Quach S, Hamid J, Pereira JA, et al. Influenza vaccination coverage across ethnic groups in Canada. CMAJ 2012;184:1673-81.

2. US Centers for Disease Control and Prevention. Final state-level influenza vaccination coverage estimates for the 2010-11 season - United States, National Immunization Survey and Behavioral Risk Factor Surveillance System, August 2010 through May 2011. Atlanta (GA): The Centers; 2011. Available: www.cdc.gov /flu/professionals/vaccination/coverage_1011estimates.htm (accessed 2012 May 16).

3. Linn ST, Guralnik JM, Patel KV. Disparities in influenza vaccine coverage in the United States, 2008. J Am Geriatr Soc 2010;58: 1333-40.

4. Uscher-Pines L, Maurer J, Harris KM. Racial and ethnic disparities in uptake and location of vaccination for 2009-H1N1 and seasonal influenza. Am J Public Health 2011;101:1252-5.

5. Brien S, Kwong JC, Buckeridge DL. The determinants of 2009 pandemic A/H1N1 influenza vaccination: a systematic review. Vaccine 2012;30:1255-64.

6. Bardenheier B, Wortley P, Ahmed F, et al. Racial inequities in receipt of influenza vaccination among long-term care residents within and between facilities in Michigan. Med Care 2011;49: $371-7$.

7. Jacobson VJ, Szilagyi PG. Patient reminder and recall systems to improve immunization rates [review]. Cochrane Database Syst Rev 2005;(3):CD003941.

8. Thomas RE, Russell M, Lorenzetti D. Interventions to increase influenza vaccination rates of those 60 years and older in the community [review]. Cochrane Database Syst Rev 2010;(9):CD005188.

9. World Health Organization. Influenza vaccines. Wkly Epidemiol $\operatorname{Rec} 2005 ; 80: 279-87$

10. Michiels B, Govaerts F, Remmen R, et al. A systematic review of the evidence on the effectiveness and risks of inactivated influenza vaccines in different target groups. Vaccine 2011;29:9159-70.

11. Dlugacz Y, Fleischer A, Carney MT, et al. 2009 H1N1 vaccination by pregnant women during the 2009-10 H1N1 influenza pandemic. Am J Obstet Gynecol 2012;206:339.e1-8.

Affiliation: Bradford Gessner is with Agence de Médecine Préventive, Paris, France. 